\title{
DIFFERENTIAL GEOMETRY OF A SURFACE AT A PLANAR POINT*
}

\author{
BY \\ V. G. GROVE \\ 1. Introduction
}

We shall say that a point $O$ of a surface $S$ is a planar point of the third order if the surface has a tangent plane at $O$ and if every curve on the surface through $O$ has an inflexion $\dagger$ at $O$. It is the purpose of this paper to study the surface $S$ in the neighborhood of such a planar point from a projective point of view by making use of the osculants $\ddagger$ of Bompiani of a plane section of $S$ through an arbitrary tangent line through $O$.

Lane and Su§ have used Bompiani's osculants to study the plane sections of a surface at a non-parabolic point on the surface. In a sense every tangent line to a surface at a planar point is an asymptotic tangent.

Downs $\|$ has studied planar points of order $n$ from a metric point of view.

In the last section we discuss the loci of certain points and lines intrinsically associated with the sections of the surface through the arbitrary tangent line as this line generates a pencil. The loci so obtained are therefore covariant loci associated with the surface and not merely with the surface in its relation to the particular tangent line.

We first find a canonical power series expansion for one non-homogeneous projective coordinate in terms of the two others. A complete geometrical description will be given of the tetrahedron of reference giving rise to this canonical expansion.

\section{THE POWER SERIES EXPANSION}

Let the equation of the analytic surface $S$ be

$$
z=f(x, y) \text {. }
$$

* Presented to the Society, April 10, 1936; received by the editors October 14, 1935, and in revised form, January 11, 1936.

$\dagger$ Each branch of the curve of intersection of the surface with its tangent plane at a planar point of third order has at least four-point contact with its tangent line at the point.

$\ddagger \mathrm{E}$. Bompiani, Per lo studio proiettivo-differenziale delle singolarita, Bollettino dell' Unione Matematica, vol. 5 (1926), p. 118. Hereafter referred to as Bompiani.

$\S \mathrm{E} . \mathrm{P}$. Lane, Plane sections through an asymptotic tangent of a surface, Bulletin of the American Mathematical Society, vol. 41 (1935), pp. 285-290; B. Su, On certain quadratic cones projectively connected with a space curve and a surface, Tôhoku Mathematical Journal, vol. 38 (1933), pp. 233-244.

\| T. L. Downs, Asymptotic and principal directions at a planar point of a surface, Duke Mathematical Journal, vol. 1 (1935), pp. 316-327. 
Let the system of coordinates be so chosen that the point $O(0,0,0)$ is the planar point, and $z=0$ the equation of the tangent plane to $S$ at $O$. The Taylor's expansion of the function $f(x, y)$ in the neighborhood of $x=0, y=0$ will be of the form

$$
z=A_{3}(x, y)+A_{4}(x, y)+A_{5}(x, y)+\cdots,
$$

wherein

$$
\begin{aligned}
& A_{3}(x, y)=a_{30} x^{3}+a_{21} x^{2} y+a_{12} x y^{2}+a_{03} y^{3} \\
& A_{4}(x, y)=a_{40} x^{4}+a_{31} x^{3} y+a_{22} x^{2} y^{2}+a_{13} x y^{3}+a_{04} y^{4}, \\
& A_{5}(x, y)=a_{50} x^{5}+a_{41} x^{4} y+a_{32} x^{3} y^{2}+a_{23} x^{2} y^{3}+a_{14} x y^{4}+a_{05} y^{5},
\end{aligned}
$$

The tangent plane $z=0$ at $O$ intersects the surface in a curve with a triple point at $O$. The triple point tangents are given by

$$
A_{3}(x, y)=0 \text {. }
$$

The equation $A_{3}(x, y)=0$ defines a cubic involution in the pencil of tangent lines to the surface at $O$, the triple lines being the triple point tangents to the curve of intersection of the surface $S$ with its tangent plane at $O$. We shall assume that the tangent line $y=z=0$ is not one of the triple lines of the cubic involution defined by $A_{3}(x, y)=0$. It follows that $a_{30} \neq 0$.

We propose first to derive a canonical form of the expansion (1) in which the edge $y=z=0$ of the tetrahedron of reference giving rise to the expansion is an arbitrary tangent line to the surface $S$ at $O$ except for the limitation noted above.

If we make the transformation

$$
x=x^{\prime}+\mu y^{\prime}, \quad y=y^{\prime}, \quad z=z^{\prime},
$$

on equation (1), and denote the new coefficients by $\bar{a}_{30}, \bar{a}_{21}, \cdots$, we find that

$$
\begin{aligned}
& \bar{a}_{30}=a_{30}, \quad \bar{a}_{21}=3 \mu a_{30}+a_{21}, \quad \bar{a}_{12}=3 \mu^{2} a_{30}+2 \mu a_{21}+a_{12}, \\
& \bar{a}_{40}=a_{40}, \quad \bar{a}_{31}=4 \mu a_{40}+a_{31}, \quad \bar{a}_{50}=a_{50}, \\
& \bar{a}_{41}=5 \mu a_{50}+a_{41}, \quad \bar{a}_{60}=a_{60}, \cdots .
\end{aligned}
$$

Hence if we choose $\mu$ to satisfy the equation

$$
3 \mu a_{30}+a_{21}=0,
$$

we may make $\bar{a}_{21}=0$. We shall assume that this transformation has been effected on the series (1).

We may give a simple geometrical characterization of the tangent line $x=z=0$ for the case in which $a_{21}=0$. The unique line of the involution defined 
by $A_{3}(x, y)=0$ corresponding to the line $y=z=0$, considered as a double line of the involution, is the line $x=z=0$ if and only if $a_{21}=0$.

The transformation

$$
x=\frac{x^{\prime}+\alpha z^{\prime}}{1+L x^{\prime}+M y^{\prime}}, \quad y=\frac{y^{\prime}+\beta z^{\prime}}{1+L x^{\prime}+M y^{\prime}}, \quad z=\frac{z^{\prime}}{1+L x^{\prime}+M y^{\prime}},
$$

does not change the edges $x=z=0, y=z=0$ of the coordinate system, and does not affect the coefficients of the form $A_{3}(x, y)$. If again we denote the new coefficients by $\bar{a}_{40}, \bar{a}_{31}, \cdots$, we find that

$$
\begin{aligned}
& \bar{a}_{40}=-2 L a_{30}+a_{40}, \bar{a}_{31}=-2 M a_{30}+a_{31}, \\
& \bar{a}_{50}=3 \alpha a_{30}^{2}-3 L a_{40}+a_{50}, \\
& \bar{a}_{41}=2 \beta a_{30} a_{12}-3 M a_{40}+a_{41}, \\
& \bar{a}_{05}=3 \beta a_{03}^{2}-3 M a_{04}+a_{05}, \cdots .
\end{aligned}
$$

We note from (8), that, with proper choice of $\alpha, L, M$, we may make $\bar{a}_{40}=\bar{a}_{31}=\bar{a}_{50}=0$. If $a_{12} \neq 0$, we may choose $\beta$ in such a way that $\bar{a}_{41}=0$. If $a_{12}=0$, we may choose $\beta$ so that $\bar{a}_{05}=0$.

The Hessian $H$ of the form

$$
A_{3}(x, y)=a_{30} x^{3}+a_{12} x y^{2}+a_{03} y^{3}
$$

is readily shown to be

$$
H=4\left[3 a_{30} a_{12} x^{2}+9 a_{30} a_{03} x y-a_{12}^{2} y^{2}\right] .
$$

Hence the tangent line $y=z=0(x=z=0)$ is one of the Hessian or neutral lines of the cubic involution determined by $A_{3}(x, y)=0$ if and only if $a_{12}=0$.

We shall say that $y=z=0$ is a Hessian tangent if $a_{12}=0$.

Let us suppose first that $y=z=0$ is not a Hessian tangent, and that the transformation (6) has been used to make $a_{40}=a_{31}=a_{50}=a_{41}=0$.

The transformation

$$
x=\frac{x^{\prime}}{1+N z^{\prime}}, \quad y=\frac{y^{\prime}}{1+N z^{\prime}}, \quad z=\frac{z^{\prime}}{1+N z^{\prime}},
$$

does not change the edges $x=z=0, y=z=0$ of the tetrahedron of reference, and does not affect the coefficients of the forms $A_{3}(x, y), A_{4}(x, y), A_{5}(x, y)$. The new coefficient $\bar{a}_{60}$ is given by the formula

$$
\bar{a}_{60}=-2 N a_{30}+a_{60} .
$$

Hence we may choose $N$ in the transformation (10) in such a manner as to make $\bar{a}_{60}=0$. We shall assume that this transformation has been effected. 
If we change the unit point by the transformation

$$
x=p x^{\prime}, \quad y=q y^{\prime}, \quad z=r z^{\prime},
$$

we find that, if $a_{22} \neq 0$, we may make $a_{30}=a_{12}=a_{22}=1$, by choosing $p, q, r$ to have the values

$$
p=a_{12} / a_{22}, \quad q= \pm\left(a_{12} a_{30}\right)^{1 / 2} / a_{22}, \quad r=a_{12}^{3} a_{30} / a_{22}^{3} .
$$

Hence the power series expansion (1) may be written in the canonical form

$$
z=\alpha_{3}(x, y)+\alpha_{4}(x, y)+\alpha_{5}(x, y)+\cdots
$$

wherein

$$
\begin{aligned}
& \alpha_{3}(x, y)=x^{3}+x y^{2}+A y^{3}, \\
& \alpha_{4}(x, y)=x^{2} y^{2}+a_{13} x y^{3}+a_{04} y^{4} \\
& \alpha_{5}(x, y)=B x^{3} y^{2}+a_{23} x^{2} y^{3}+a_{14} x y^{4}+a_{05} y^{5} \\
& \alpha_{6}(x, y)=C x^{5} y+\cdots, \quad \alpha_{7}(x, y)=D x^{7}+E x^{6} y+\cdots, \\
& \alpha_{8}(x, y)=F x^{8}+\cdots, \quad \alpha_{9}(x, y)=G x^{9}+\cdots .
\end{aligned}
$$

The edge $y=z=0$ is an arbitrary tangent line, not a tangent line to the curve of intersection of the surface with its tangent plane, and not a Hessian tangent. Every coefficient of the canonical expansion (13) is an absolute invariant expressing the relation between the surface $S$ at the planar point $O$ and the tangent line $y=z=0$. In the expressions (14) we have written only those terms which will be of use in the discussions to follow.

If $y=z=0$ is a Hessian tangent we may, as was remarked previously, make use of the coefficient $\beta$ of the transformation (7) to make $a_{05}=0$. We may as before choose the coefficient $N$ of the transformation (10) to make $a_{60}=0$. If the two Hessian tangents are not coincident, and if $a_{22} \neq 0$, by proper choice of the unit point we may make $a_{30}=a_{03}=a_{22}=1$. Hence the power series expansion (1) may be written in the canonical form

$$
\begin{aligned}
z= & x^{3}+y^{3}+x^{2} y^{2}+A^{\prime} x^{4} y+\cdots+B^{\prime} x^{5} y+\cdots \\
& +C^{\prime} x^{7}+D^{\prime} x^{6} y+\cdots+E^{\prime} x^{8}+\cdots+F^{\prime} x^{9}+\cdots .
\end{aligned}
$$

The edges $y=z=0, x=z=0$ of the tetrahedron of reference are the Hessian tangent to $S$ at $O$. Every coefficient of the expansion (15) is an absolute invariant of the surface.

\section{Plane sections through a tangent line}

Let us first consider a surface $S$ whose equation in non-homogeneous projective coordinates $(x, y, z)$ has been reduced to the form (13). Let us intro- 
duce homogeneous projective coordinates $\left(x_{1}, x_{2}, x_{3}, x_{4}\right)$ of a point whose nonhomogeneous projective coordinates are $(x, y, z)$ by placing

$$
x=\frac{x_{2}}{x_{1}}, \quad y=\frac{x_{3}}{x_{1}}, \quad z=\frac{x_{4}}{x_{1}} .
$$

The plane whose equation is

$$
y=n z, \quad n \neq 0,
$$

cuts the surface in a curve $\Gamma$ whose projection $C$ onto the tangent plane from $\mathrm{O}_{3}(0,0,0,1)$ has the equations

$$
\begin{aligned}
& y=n\left[x^{3}+x y^{2}+A y^{3}+x^{2} y^{2}+\cdots+B x^{3} y^{2}+\cdots+C x^{5} y+\cdots\right], \\
& z=0 .
\end{aligned}
$$

If we solve the series in (17) for $y$ as a power series in $x$ we obtain

$$
y=n\left[x^{3}+b_{7} x^{7}+b_{8} x^{8}+b_{9} x^{9}+\cdots\right]
$$

wherein

$$
b_{7}=n^{2}+D, \quad b_{8}=n^{2}+C n+F, \quad b_{9}=A n^{3}+B n^{2}+E n+G .
$$

Equations (16) and (18) are the equations of an arbitrary plane section $\Gamma$ of the surface $S$ through an arbitrary tangent line not a Hessian tangent at a planar point $O$ of $S$.

Suppose next that the tangent line $y=z=0$ is a Hessian tangent line, and that the equation of the surface has been reduced to the form (15). The plane (16) intersects the surface $S$ in a curve $\Gamma$ whose projection $C$ onto the tangent plane from $O_{3}(0,0,0,1)$ has the equations

$$
y=n\left[x^{3}+c_{7} x^{7}+c_{8} x^{8}+c_{9} x^{9}+\cdots\right], \quad z=0,
$$

wherein

$$
c_{7}=A^{\prime} n+C^{\prime}, \quad c_{8}=n^{2}+B^{\prime} n+E^{\prime}, \quad c_{9}=n^{3}+D^{\prime} n+F^{\prime} .
$$

\section{Bompiani's OSCULANTS AND THEIR LOCI}

We may use the expansions (18) and (20) to derive the equations of the osculants used by Bompiani in discussing the various neighborhoods of an inflexion of a plane curve. We observe first that the expansions (18) and (20) are identical in form with the expansion* (2) of Bompiani's paper.

Let us first discuss the equations (18) and $z=0$. These are the equations of the projection $C$ of the curve $\Gamma$ of intersection of the plane (16) with the surface $S$.

* Bompiani, p. 119. 
The equations of the cusped cubic with seven-point contact with $C$ at $O$ are

$$
y=n x^{3}, \quad z=0 .
$$

This cubic has of course an inflexion at $O(1,0,0,0)$ and its cusp is at the point $\mathrm{O}_{2}(0,0,1,0)$. The cusp tangent of the cubic is the line $x_{1}=x_{4}=0$. The locus of the cusps for all plane sections $\Gamma$ through $y=z=0$ is therefore a line, namely, the edge $x_{1}=x_{2}=0$ of the tetrahedron of reference. The cusp locus of all six-point cusped cubics of the curve $C$ is the line $x=z=0$ previously characterized as the line corresponding to the line $y=z=0$ considered as a double line in the cubic involution in the pencil of tangent lines determined by $\alpha_{3}(x, y)=0$. The cusp tangent intersects the tangent $y=z=0$ at the point $O_{1}(0,1,0,0)$ of the tetrahedron of reference. The edges $x_{1}=x_{2}=0, x_{1}=x_{4}=0$ and the vertices $O_{1}$ and $O_{2}$ of the tetrahedron of reference giving rise to the canonical expansion (13) are therefore characterized geometrically.

The locus of the cusped cubics for all plane sections $\Gamma$ is the cubic cone

$$
z=x^{3}
$$

with vertex at the cusp $\mathrm{O}_{2}$ of the seven-point cusped cubic (22).

The equations of the cubic having eight-point contact with $\Gamma$ at $O$ and passing through the cusp of the seven-point cusped cubic of $\Gamma$ are

$$
n y=n^{2} x^{3}+b_{7} x y^{2}, \quad y=n z .
$$

The locus of the curve (24) for all sections through the given arbitrary tangent line is the algebraic surface of order three whose equation is

$$
z=x^{3}+x\left(y^{2}+D z^{2}\right) .
$$

The surfaces (23) and (25) intersect in the line $x=z=0$, and in two plane curves whose projections from the point $O_{3}$ onto the tangent plane are the cusped cubics

$$
y= \pm(-D)^{1 / 2} x^{3}, \quad z=0,
$$

and whose projections from the point $O_{1}$ onto the face of the tetrahedron opposite $O_{1}$ are the straight lines

$$
y^{2}+D z^{2}, \quad x=0 .
$$

The equations of the nine-point quartic curve passing through the point $O_{1}(0,1,0,0)$, tangent to the cusp tangent $x_{1}=x_{4}=0$, having a node at the point $O_{2}(0,0,1,0)$ with the cusp locus $x_{2}=x_{4}=0$ for one nodal tangent are

$$
n y=n^{2} x^{3}+b_{7} x y^{2}+b_{8} x^{2} y^{2}, \quad z=0 .
$$


The residual nodal tangent of the quartic (26) is the line having the equations

$$
b_{7} x_{1}+b_{8} x_{2}=0, \quad x_{4}=0 .
$$

The locus of the nine-point quartic curve of the section $\Gamma$ for all sections $\Gamma$ is the quartic surface whose equation is

$$
z=x^{3}+x\left(y^{2}+D z^{2}\right)+x^{2}\left(y^{2}+C y z+F z^{2}\right) .
$$

The locus of the residual nodal tangent of the nine-point quartics of the sections $\Gamma$ is the cubic surface

$$
y^{2}+D z^{2}+x\left(y^{2}+C y z+F z^{2}\right)=0 .
$$

The locus of the cusps $x_{1}=x_{2}=0$ of the seven-point cusped cubics lies on the cubic surface (29). The tangent plane to the surface (29) at the point $(0,0, \lambda, \mu)$ is the plane

$$
\left(\lambda^{2}+D \mu^{2}\right) x_{1}+\left(\lambda^{2}+C \lambda \mu+F \mu^{2}\right) x_{2}=0 .
$$

The tangent plane (30) coincides with the plane determined by the locus of the cusps and by $O$ if and only if

$$
\lambda^{2}+D \mu^{2}=0 .
$$

There exist therefore two points $P_{1}, P_{2}$ on the locus of the cusps of the seven-point cusped cubics of plane sections through an arbitrary non-Hessian tangent at a planar point $O$ such that the tangent plane to the locus of the residual nodal tangent of the nine-point nodal quartic coincides with the plane determined by the point $O$ and the locus of the cusps of the seven-point cusped cubics. The vertex $\mathrm{O}_{3}(0,0,0,1)$ is determined on the locus of the cusps as the harmonic conjugate of the cusp $O_{2}$ with respect to the points $P_{1}, P_{2}$. The four vertices of the tetrahedron of reference giving rise to the expansion (13) have therefore been characterized geometrically. In the last section we shall characterize the unit point of the system of coordinates.

The seven-point cusped cubic and the nine-point quartic of the projection $C$ of $\Gamma$ intersect at $O$ and also at the point

$$
\left(-\frac{b_{7}}{b_{8}},-\frac{n b_{7}^{3}}{b_{8}{ }^{3}}, 0\right) \text {. }
$$

The coordinates of the intersection of the seven-point cusped cubic and the nine-point quartic of the curve of section $\Gamma$ are

$$
\left(-\frac{b_{7}}{b_{8}},-\frac{n b_{7}^{3}}{b_{8}{ }^{3}},-\frac{b_{7}^{3}}{b_{8}^{3}}\right) \text {. }
$$


The locus of this point for all sections $\Gamma$ of the surface through the tangent $y=z=0$ is a rational curve of order seven tangent to $y=z=0$ at $O$.

The line through $O$ and the point (31) has the equations

$$
b_{8}^{2} y=n b \tau^{2} x, \quad y=n z .
$$

The locus of this line is the quintic cone

$$
x\left(y^{2}+D z^{2}\right)^{2}=z\left(y^{2}+C y z+F z^{2}\right)^{2} .
$$

The lines $x=z=0, y=z=0$ lie on the cone. The plane $x-z=0$ is tangent to the cone along the line $x=z=0$. The line $y=z=0$ is a singular line on the cone, the planes $y^{2}+D z^{2}=0$ being tangent to the cone along this generator.

From (18) we find readily that

$$
\begin{aligned}
& y-n x^{3}=n\left[b_{7} x^{7}+b_{8} x^{8}+\cdots\right], \\
& y-n x^{3}-\frac{b_{7}}{n} x y^{2}=n\left[b_{8} x^{8}+b_{9} x^{9}+\cdots\right], \\
& y-n x^{3}-\frac{b_{7}}{n} x y^{2}-\frac{b_{8}}{n} x^{2} y^{2}=n b_{9} x^{9}+\cdots .
\end{aligned}
$$

It follows therefore that the seven-point cusped cubic has eight-point contact with $\Gamma$ if and only if $b_{7}=0$, the eight-point cubic (24) has nine-point contact with $\Gamma$ if and only if $b_{8}=0$, and the nine-point quartic has ten-point contact with $\Gamma$ if and only if $b_{9}=0$. There are two plane sections through an arbitrary tangent line at a planar point of a surface such that the seven-point cusped cubic of the section has eight-point contact with the section. The planes of these sections separate the planes $y=0, z=0$ harmonically. The face $y=0$ of the tetrahedron of reference giving rise to the canonical expansion (13) has therefore been given a simple geometrical characterization.

Let us now consider the osculants of Bompiani for the curves of intersection of the surface $S$ by planes through a Hessian tangent line at the planar point. If use be made of equations (20), we find readily that the equations of the seven-point cusped cubic, the eight-point cubic, and the nine-point nodal quartic of the section $\Gamma$ through a Hessian tangent are respectively

$$
\begin{aligned}
y & =n x^{3}, \quad y=n z ; \\
n y & =n^{2} x^{3}+c_{7} x y^{2}, \quad y=n z ; \\
n y & =n^{2} x^{3}+c_{7} x y^{2}+c_{8} x^{2} y^{2}, \quad y=n z .
\end{aligned}
$$

The loci of these curves are respectively

$$
\begin{aligned}
& z=x^{3} \\
& z=x^{3}+x z\left(A^{\prime} y+C^{\prime} z\right) \\
& z=x^{3}+x z\left(A^{\prime} y+C^{\prime} z\right)+x^{2}\left(y^{2}+B^{\prime} y z+E^{\prime} z^{2}\right) .
\end{aligned}
$$


From (20) we find that

$$
\begin{aligned}
& y-n x^{3}=n\left[c_{7} x^{7}+c_{8} x^{8}+\cdots\right], \\
& y-n x^{3}-\frac{c_{7}}{n} x y^{2}=n\left[c_{8} x^{8}+c_{9} x^{9}+\cdots\right], \\
& y-n x^{3}-\frac{c_{7}}{n} x y^{2}-\frac{c_{8}}{n} x^{2} y^{2}=n c_{9} x^{9}+\cdots
\end{aligned}
$$

It follows that through a Hessian tangent line there is only one plane section having eight-point contact with its seven-point cusped cubic, two sections having nine-point contact with their eight-point cubics, and three sections having ten-point contact with their nine-point quartics.

The curves (35) and (37) intersect at $O$ and at the point

$$
\left(-\frac{c_{7}}{c_{8}},-\frac{c_{7}^{3}}{c_{8}^{3}},-\frac{c_{7}^{3}}{c_{8}^{3}}\right) \text {. }
$$

The locus of the line joining $O$ to this point is a quartic cone whose equation is

$$
\left(y^{2}+B^{\prime} y z+E^{\prime} z^{2}\right)^{2}=x z\left(A^{\prime} y+C^{\prime} z\right)^{2} .
$$

Other discussions similar to those made for sections of the surface through a non-Hessian tangent could be made, but we shall carry this discussion no further.

\section{LOCI RELATED TO THE SURFACE $S$ AT $O$}

The points, curves, and surfaces discussed in the previous sections have, in the main, given properties of the configuration composed of the surface $S$ and the given tangent line used in making the sections of the surface. For example, the cusp of the seven-point cusped cubic of the projection of the plane section is a point intrinsically related to the tangent line through which the plane sections were made. Likewise the locus of this point is a straight line which is related to the particular tangent line through which the sections were made.

In this section we propose to derive the necessary formulas for studying the loci of the various points, lines, and the envelopes of the surfaces derived in the previous sections as the given tangent line generates the pencil of tangent lines. Thus we obtain loci covariant to the surface $S$ at the planar point.

To the end in view we shall make the transformation

$$
x=\bar{x}, \quad y=m \bar{x}+\bar{y}, \quad z=\bar{z},
$$

on the expansion (13). We obtain an expansion of the form

$$
\bar{z}=B_{3}(\bar{x}, \bar{y})+B_{4}(\bar{x}, \bar{y})+\cdots \text {. }
$$


We now, by means of transformations similar to (4), (7), (10), and (11), reduce (44) to the canonical form resembling the form (13) except that the line $\bar{y}=\bar{z}=0$ is the arbitrary tangent line used as one edge of the tetrahedron of reference giving rise to the canonical expansion.

The forms $B_{3}(\bar{x}, \bar{y}), \cdots$ have coefficients $\bar{a}_{30}, \bar{a}_{21}, \cdots$ given by the formula

$$
\bar{a}_{j k}=\frac{1}{k !} \frac{d^{k} \alpha_{j+k}(1, m)}{d m^{k}} \quad(j+k \geqq 3) .
$$

The canonical form of the expansion (44) with $y^{\prime}=z^{\prime}=0(\bar{y}=\bar{z}=0)$ as one edge of the tetrahedron of reference is of the form

$$
z^{\prime}=\alpha_{3}^{\prime}\left(x^{\prime}, y^{\prime}\right)+\alpha_{4}^{\prime}\left(x^{\prime}, y^{\prime}\right)+\cdots .
$$

By means of (4), (7), (10), (11), and (43) the transformation of coordinates between the tetrahedron of reference used in the canonical form (13) to that of the canonical form (46) is

$$
\begin{aligned}
& \rho x_{1}=x_{1}^{\prime}+\bar{p} \bar{L} x_{2}^{\prime}+\bar{q} \bar{M} x_{3}^{\prime}+\bar{r} \bar{N} x_{4}^{\prime}, \\
& \rho x_{2}=\quad \bar{p} x_{2}^{\prime}+\bar{q} \bar{\mu} x_{3}^{\prime}+\bar{r}(\bar{\alpha}+\bar{\beta} \bar{\mu}) x_{4}^{\prime}, \\
& \rho x_{3}=\quad \bar{m} m x_{2}^{\prime}+\bar{q}(1+m \bar{\mu}) x_{3}^{\prime}+\bar{r}[\bar{\beta}+m(\bar{\alpha}+\bar{\beta} \bar{\mu})] x_{4}^{\prime}, \\
& \rho x_{4}= \\
& \bar{r} x_{4}^{\prime},
\end{aligned}
$$

wherein $\bar{\alpha}, \bar{\beta}, \cdots$ are defined by formulas used in reducing (1) to the form (13) but with $a_{j k}$ replaced by $\bar{a}_{j k}$ defined by (45). These formulas are

$$
\begin{aligned}
& 3 \bar{a}_{30} \bar{\mu}+\bar{a}_{21}=0, \quad 2 \bar{a}_{30} \bar{L}=\bar{a}_{40}, \\
& 2 \bar{a}_{30} \bar{M}=\bar{a}_{31}+4 \bar{a}_{40} \bar{\mu}, \quad 3 \bar{\alpha} \bar{a}_{30}^{2}=3 \bar{L} \bar{a}_{40}-\bar{a}_{50}, \\
& 2 \bar{a}_{30} \bar{\beta}\left[3 \bar{a}_{30} \bar{\mu}^{2}+2 \bar{a}_{21} \bar{\mu}+\bar{a}_{12}\right]=3 \bar{M} \bar{a}_{40}-\bar{a}_{41}-5 \bar{\mu} \bar{a}_{50}, \\
& 2 \bar{a}_{30} \bar{N}=\bar{a}_{60} .
\end{aligned}
$$

Denote by $O, \mathrm{O}_{1}^{\prime}, \mathrm{O}_{2}^{\prime}, \mathrm{O}_{3}^{\prime}$ the vertices of the tetrahedron giving rise to the canonical expansion (46) in such a manner that $O_{1}^{\prime}, O_{2}^{\prime}, O_{3}^{\prime}$ have geometrical characterizations similar to $O_{1}, O_{2}, O_{3}$.

By means of (47) and (48) we find readily that the parametric equations of the locus of the point $O_{1}^{\prime}$ as the line $y^{\prime}=z^{\prime}=0$ generates the pencil of tangent lines are

$$
\begin{aligned}
& x_{1}=m^{2}+a_{13} m^{3}+a_{04} m^{4}, \\
& x_{2}=2\left(1+m^{2}+A m^{3}\right), \\
& x_{3}=2 m\left(1+m^{2}+A m^{3}\right), \\
& x_{4}=0 .
\end{aligned}
$$


The locus of $O_{1}^{\prime}$ of course passes through the point $O_{1}$. Moreover the tangent line to the curve at $O_{1}$ is the line $x_{1}=x_{4}=0$. Hence the envelope of the cusp tangents of the seven-point cusped cubics of the projections $C$ of all sections of a surface at a planar point of the third order is a rational quartic curve. It follows also that the plane $O_{1}^{\prime}, O_{2}^{\prime}, O_{3}^{\prime}$ is tangent at $O_{1}^{\prime}$ to the ruled surface generated by the line $O_{1}^{\prime} O_{3}^{\prime}$ as the tangent $y^{\prime}=z^{\prime}=0$ describes the pencil of tangent lines at the planar point $O$.

If we eliminate $m$ homogeneously from the parametric equations of the locus of $O_{1}^{\prime}$, we may write the equations of the locus of $O_{1}^{\prime}$ in the form

$$
2\left(x^{3}+x y^{2}+A y^{3}\right)=x^{2} y^{2}+a_{13} x y^{3}+a_{04} y^{4}, \quad z=0 .
$$

Hence the locus of $O_{1}^{\prime}$ has a triple point at $O$, the triple point tangents being the tangents to the curve of intersection of the surface with its tangent plane at $O$.

Equation (50) furnishes a rather simple interpretation of the form $\alpha_{4}(x, y)$. The line $x_{1}=x_{4}=0$ intersects the quartic (50) in four points. The equations of the four lines through $O$ and these four points are

$$
\alpha_{4}(x, y)=0, \quad z=0 .
$$

The equations of the conic tangent to the line $y=z=0$ at $O$ and having three-point contact at $O_{1}$ with the curve (50) are

$$
x_{3}^{2}-2 x_{1} x_{2}=0, \quad x_{4}=0 .
$$

The parametric equations of the locus of the point $O_{2}^{\prime}$ as $y^{\prime}=z^{\prime}=0$ generates the pencil of tangent lines are readily found to be

$$
\begin{aligned}
x_{1}= & 3\left(1+m^{2}+A m^{3}\right)\left(2 m+3 a_{13} m^{2}+4 a_{04} m^{3}\right) \\
& -4\left(2 m+3 A m^{2}\right)\left(m^{2}+a_{13} m^{3}+a_{04} m^{4}\right), \\
x_{2}= & -2\left(1+m^{2}+A m^{3}\right)\left(2 m+3 A m^{2}\right), \\
x_{3}= & 2\left(1+m^{2}+A m^{3}\right)\left(m^{2}+3\right), \\
x_{4}= & 0 .
\end{aligned}
$$

The locus of the cusp $\mathrm{O}_{2}^{\prime}$ of the seven-point cusped cubic is therefore a rational quintic curve. This curve has a triple point at $O$, the triple point tangents being the tangents to the curve of intersection of the surface with its tangent plane at $O$. The tangent to the curve (52) at $\mathrm{O}_{2}$ has the equations

$$
2 x_{1}+3 x_{2}=0, \quad x_{4}=0 .
$$

We have already characterized the vertices of the tetrahedron of reference giving rise to the expansion (13). We may characterize the unit point in the following manner. First the line (53) and the edges $x_{1}=x_{4}=0, x_{2}=x_{4}=0$ en- 
able us to characterize the line $x_{1}-x_{2}=x_{4}=0$ by means of a cross ratio. This latter line intersects the conic (51) in two points $Q_{1}$ and $Q_{2}$. Either of these two points and the points $O_{1}, O_{2}$ may be used to characterize the line $x_{2}-x_{3}=x_{4}=0$ by means of a cross ratio. The choice of either $Q_{1}$ or $Q_{2}$ corresponds to the choice of sign in the double sign of $q$ in (12). The point $E(1,1,1,0)$ is therefore characterized. The line $E O_{3}$ intersects the cone(23) in the unit point.

Michigan State College,

East Lansing, Mich. 